\title{
Mortalidade devido a acidentes de bicicletas em Pernambuco, Brasil
}

\author{
Mortality due to bicycle accidents in Pernambuco, Brazil
}

Pauliana Valéria Machado Galvão ${ }^{1}$

Luciana Pinto Pestana ${ }^{1}$

Valter Mário Pestana ${ }^{1}$

Michelline Oliveira Pedrosa Spíndola ${ }^{1}$

Reginaldo Inojosa Carneiro Campello ${ }^{1}$

Eliane Helena Alvim de Souza ${ }^{1}$

${ }^{1}$ Faculdade de Odontologia,

Abstract The scope of this paper was to conduct a quantitative analysis of deaths resulting from bicycle accidents in the state of Pernambuco by studying secondary data between 2001 and 2010. The sample consisted of all the Deaths recorded in the Mortality Information System of the Unified Health System Database that reported bicycle accidents between 2001 and 2010. Descriptive measures were determined for all variables. Socio-demographic variables were paired with the basic cause of death in order to find a statistical correlation. In Pernambuco, the aforementioned information system recorded 517 deaths resulting from bicycle accidents, with greater frequency in men between 25 and 59 years of age, AfroBrazilians, single and of unknown schooling. The mean age was 36.82 years $(S D=17.026)$, and the minimum and maximum age of 4 and 86 years old, respectively. The findings highlight the need for the creation of adequate infrastructure and effective legal measures to prevent traffic accidents involving this type of vehicle, relying on the evidence of distribution of cases in most Pernambuco municipalities.

Key words Traffic accidents, Cycling, External causes, Mortality
Resumo $O$ artigo tem por objetivo analisar os casos de morte nos acidentes com bicicleta ocorridos em Pernambuco entre 2001 e 2010. Este estudo quantitativo analisou dados secundários. A amostra consistiu de todas as Declarações de Óbitos registradas no Sistema de Informação de Mortalidade do Banco de Dados do Sistema Único de Saúde que relataram acidentes de bicicleta entre 2001 e 2010. Medidas descritivas foram determinadas para todas as variáveis. As sociodemográficas foram cruzadas com a causa básica de morte em busca de correlação estatística. Em Pernambuco, no citado sistema de informação, foram registradas $517 \mathrm{De}$ clarações de Óbitos decorrentes de acidentes de bicicleta, sendo nestes sinistros a participação mais frequente de homens, entre 25-59 anos, pardos, solteiros e de escolaridade ignorada. A idade média foi de 36,82 anos (desvio padrão $=17,026$ ), sendo a idade mínima e a máxima, respectivamente, 4 e 86 anos. Os achados apontam para a necessidade da criação de infraestrutura adequada e de medidas legais efetivas para prevenir acidentes de tráfego envolvendo este tipo de veículo, apoiando-se na evidência de distribuição de casos na maioria dos municípios de Pernambuco.

Palavras-chave Acidentes de trânsito, Ciclismo, Causas Externas, Mortalidade
Universidade de

Pernambuco. Av. Agamenon Magalhães S/N, Santo Amaro. 50100-010 Recife PE.e.ha.souza@hotmail.com 


\section{Introdução}

No mundo, cerca de 1,2 milhões de pessoas morrem todos os anos nas estradas, entre 20 e 50 milhões sofrem injúrias não fatais e cerca de $90 \%$ das fatalidades do trânsito ocorrem em países subdesenvolvidos ou em desenvolvimento, como o Brasil ${ }^{1}$.

O Brasil ocupa o quinto lugar no ranking mundial de mortalidade para este tipo de lesão e os usuários mais vulneráveis do sistema viário em termos de exposição corporal são pedestres, ciclistas e motociclistas ${ }^{2}$. Atualmente, é a sexta maior frota de bicicletas do mundo, estimada em 75 milhões de unidades, sendo o quarto maior fabricante, atrás de China, Índia e Alemanha. Apesar disto, conta com somente três mil quilômetros de infraestrutura cicloviária ${ }^{3}$.

A bicicleta é um veículo considerado prático, barato e favorável ao meio ambiente e seu uso é particularmente popular entre crianças pequenas e adolescentes ${ }^{4}$. Algumas das outras razões para esta popularidade são o preço da gasolina, uma população cada vez mais móvel ${ }^{5}$ e a visão da bicicleta como um tipo de equipamento de esporte de alta tecnologia e um meio saudável de transporte ${ }^{6}$. No entanto sua utilização quer para transporte, atividades recreativas ou desportivas, não está isenta de acidentes ${ }^{7}$ havendo poucos estudos sobre o seu perfil de uso e suas consequências ${ }^{8}$.

Uma das principais características do transporte por ciclovias é a sua flexibilidade em áreas urbanas. A bicicleta é disponível em quase todas as partes da cidade, não necessitando de espaço físico grande, embora sua rota seja limitada a $7,5 \mathrm{~km}$, distância considerada confortável devido

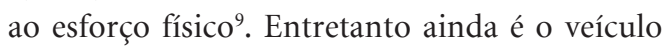
não motorizado mais comumente envolvido em acidentes ${ }^{4}$.

Carente de estudos nacionais sistematizados, Pernambuco não tem a real dimensão da prevalência da mortalidade por acidentes de bicicleta. Este estudo foi desenvolvido a fim de medir a prevalência de mortes em acidentes de bicicletas em uma série de 10 anos a partir de 2001.

\section{Métodos}

Este estudo foi descritivo e analítico, quantitativo, através de dados secundários. Todas as Declarações de Óbitos (DO) registradas no Banco de Dados do Sistema de Informações sobre Mortalidade (SIM, DATASUS) entre 2001 e 2010 foram estudadas.
Os arquivos relativos a esses anos foram adquiridos pela internet e os dados foram tratados no programa Excel (versão 2010) e analisados estatisticamente com o Programa Statistical Package for Social Services (SPSS versão 13.0).

As causas básicas de óbito trabalhadas foram: Colisão com Pedestre ou Animal (CPA); Colisão com Outro Veículo a Pedal (COVP); Colisão com Veículo Motorizado de 2-3 Rodas (CVM2-3R); Colisão com Automóvel, Caminhonete ou Caminhão (CACC); Colisão com Transporte Pesado ou Ônibus (CTPO); Colisão com Trem ou Veículo Ferroviário (CTVF); Colisão com Outro Veículo Não-Motorizado (COVNM); Colisão com Objeto Fixo ou Estacionário (COFE); Acidentes Não de Trânsito (ANDT); Acidente Inespecífico (AI).

As variáveis sociodemográficas (sexo, faixa etária, raça, estado civil e escolaridade) e a causa básica de óbito foram cruzadas e analisadas com teste qui-quadrado de Pearson ou teste exato de Fisher, quando o primeiro foi impraticável. Medidas de tendência central (mediana, média e moda) foram identificadas para a idade absoluta.

A distribuição espacial utilizou a divisão administrativa disponível no Website do Instituto Brasileiro de Geografia e Estatística ${ }^{10}$, onde o Estado de Pernambuco é dividido em 5 mesorregiões de acordo com características socioeconômicas:

. Região Metropolitana - consiste em 14 municípios e o Arquipélago de Fernando de Noronha;

. Mata Pernambucana - consiste em $43 \mathrm{mu}$ nicípios, localizada na faixa litorânea em torno da Região Metropolitana, se estendendo até os limites do Agreste;

- Agreste Pernambucano - consiste em 69 municípios, situados na Região do Planalto da Borborema;

. São Francisco Pernambucano - consiste em 15 municípios;

. Sertão Pernambucano: consiste em $41 \mathrm{mu}$ nicípios, sendo a mesorregião de menor densidade demográfica.

\section{Resultados}

No período considerado foram levantadas 517 Declarações de Óbitos onde as mortes foram consequência de acidentes com bicicleta em Pernambuco, sendo mais frequentes em homens, entre 25-39 anos e 40-59 anos, pardos, solteiros e de escolaridade ignorada. A idade média foi de 
36,82 anos, a mediana foi de 34 anos, a moda foi de 21 e o desvio padrão (DP) foi igual a 17,026 (Tabela 1) e a variação da idade compreendeu de 4 a 86 anos.

As maiores casuísticas foram encontradas nos anos de 2009 (64 óbitos) e 2007 (61 óbitos), equivalendo a $12,4 \%$ e $11,8 \%$ do total de casos encontrados. As menores ocorreram em 2005 e 2004 (representando, respectivamente, 6,8\% e 7,7\% do total) (Figura 1).

Tabela 1. Características sociodemográficas das vítimas fatais devido a acidentes com ciclistas/ bicicletistas, Pernambuco, 2001-2010.

\begin{tabular}{|c|c|c|}
\hline Variável & $\mathbf{N}$ & $\%$ \\
\hline \multicolumn{3}{|l|}{ Gênero } \\
\hline Masculino & 481 & 93,0 \\
\hline Feminino & 36 & 7,0 \\
\hline Total & 517 & 100,0 \\
\hline \multicolumn{3}{|l|}{ Grupo Etário $^{1}$} \\
\hline $0-11$ anos & 21 & 4,1 \\
\hline $12-17$ anos & 36 & 7,0 \\
\hline $18-24$ anos & 83 & 16,1 \\
\hline $25-39$ anos & 163 & 31,5 \\
\hline $40-59$ anos & 150 & 29,0 \\
\hline 60 anos ou mais & 64 & 12,4 \\
\hline Total & 517 & 100,0 \\
\hline \multicolumn{3}{|l|}{ Raça } \\
\hline Branco & 95 & 18,4 \\
\hline Preto & 16 & 3,1 \\
\hline Pardo & 377 & 72,9 \\
\hline Amarelo & 1 & 0,2 \\
\hline Indígena & 2 & 0,4 \\
\hline Não Informado & 26 & 14,7 \\
\hline Total & 517 & 100,0 \\
\hline \multicolumn{3}{|l|}{ Estado Civil } \\
\hline Solteiro & 287 & 55,5 \\
\hline Casado & 161 & 31,1 \\
\hline Ignorado & 41 & 7,9 \\
\hline Não Informado & 28 & 5,4 \\
\hline Total & 517 & 100,0 \\
\hline \multicolumn{3}{|l|}{ Escolaridade } \\
\hline Nenhuma & 67 & 13,0 \\
\hline $1-3$ anos & 82 & 15,9 \\
\hline $4-7$ anos & 87 & 16,8 \\
\hline $8-11$ anos & 34 & 6,6 \\
\hline 12 anos ou mais & 11 & 2,1 \\
\hline Ignorado & 160 & 48,7 \\
\hline Não Informado & 76 & 14,7 \\
\hline Total & 517 & 100,0 \\
\hline
\end{tabular}

${ }^{1}$ Média $=36,82 ;$ Mediana $=34 ;$ Moda $=21 ; \mathrm{DP}=17,026$ Fonte: SIM/ Banco de Dados do Sistema Único de Saúde/ Ministério da Saúde, 2012.
A causa básica mais prevalente foi acidente inespecífico, onde a vítima era um ciclista, mas nenhuma informação adicional sobre o acidente foi relacionada. A causa jurídica da morte está listada como acidente predominantemente. O local mais frequente do óbito foi a via pública, indicando que a maioria das vítimas não sobrevivem ao resgate. A necropsia foi realizada em $59,4 \%$ dos casos. Geralmente, a fonte de informação é o Boletim de Ocorrência (Tabela 2).

A distribuição espacial das mortes demonstra uma concentração maior dos óbitos na Região Metropolitana, seguida da região Agreste (Figura 2). Dos 182 municípios que formam o estado, 116 relataram ao longo destes 10 anos acidentes com bicicletas que tiveram vítimas fatais, correspondendo a 63,7\% dos municípios (dados não tabulados).

As relações entre a causa básica da morte e as características sociodemográficas não demonstraram significância estatística e mostraram o mesmo padrão de distribuição destas, exceto para a escolaridade e causa básica "colisão com objeto fixo ou estacionário", na qual predominou na escolaridade de 4-7 anos (30,8\%). Entretanto, outros fatos ficaram evidentes sobre acidentes com bicicleta e o grupo etário envolvido (Tabela 3):

. São raras as ocorrências de acidentes envolvendo bicicletas e outros veículos não motorizados;

. Os acidentes inespecíficos foram os mais referidos em todas as faixas etárias;

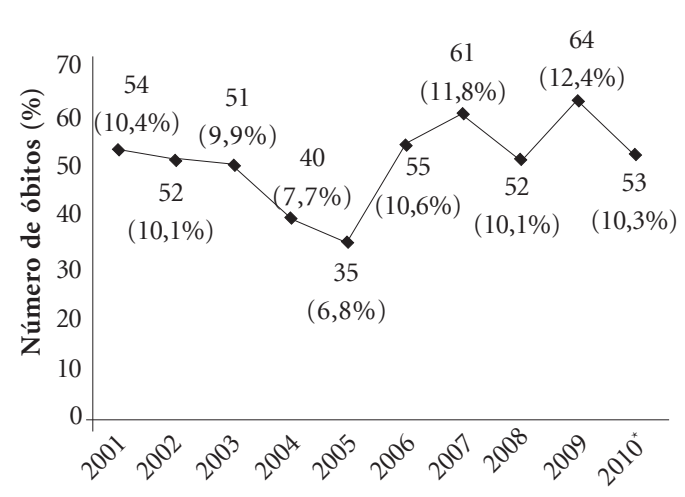

Figura 1. Distribuição temporal de óbitos devido a acidentes com ciclistas/bibicletistas, Pernambuco, 2001-2010.

${ }^{*}$ dados preliminares

Fonte: SIM/Banco de Dados do Sistema Único de Saúde/ Ministério da Saúde, 2012. 
Tabela 2. Causa Básica e outros dados de óbitos devido a acidentes com ciclistas/bicicletistas, Pernambuco, 2001-2010.

\begin{tabular}{|c|c|c|}
\hline Variável & $\mathbf{N}$ & $\%$ \\
\hline \multicolumn{3}{|l|}{ Causa Básica de Morte } \\
\hline $\begin{array}{l}\text { Colisão com pedestre ou animal } \\
\text { (CPA) }\end{array}$ & 13 & 2,5 \\
\hline $\begin{array}{l}\text { Colisão com outro veículo a pedal } \\
\text { (COVP) }\end{array}$ & 14 & 2,7 \\
\hline $\begin{array}{l}\text { Colisão com veículo a motor de } 2-3 \\
\text { rodas (CVM2-3R) }\end{array}$ & 40 & 7,7 \\
\hline $\begin{array}{l}\text { Colisão com automóvel, } \\
\text { caminhonete ou caminhão } \\
\text { (CACC) }\end{array}$ & 102 & 19,7 \\
\hline $\begin{array}{l}\text { Colisão com transporte pesado ou } \\
\text { ônibus (CTPO) }\end{array}$ & 90 & 17,4 \\
\hline $\begin{array}{l}\text { Colisão com outro veículo não } \\
\text { motorizado (COVNM) }\end{array}$ & 2 & 0,4 \\
\hline $\begin{array}{l}\text { Colisão com objeto fixo ou } \\
\text { estacionário (COFE) }\end{array}$ & 13 & 2,5 \\
\hline Acidente não-de-trânsito (ANDT)* & 94 & 18,2 \\
\hline Acidente inespecífico (AI) & 149 & 28,8 \\
\hline Total & 517 & 100,0 \\
\hline \multicolumn{3}{|l|}{ Causa jurídica de morte } \\
\hline Acidente & 503 & 97,3 \\
\hline Desconhecido & 14 & 2,7 \\
\hline Total & 517 & 100,0 \\
\hline \multicolumn{3}{|l|}{ Local de Ocorrência de Morte } \\
\hline Hospital & 211 & 40,8 \\
\hline Outro estabelecimento de morte & 1 & 0,2 \\
\hline Residência & 2 & 0,4 \\
\hline Via pública & 270 & 52,2 \\
\hline Outros & 33 & 6,3 \\
\hline Total & 517 & 100,0 \\
\hline \multicolumn{3}{|l|}{ Realização de Necrópsia } \\
\hline Sim & 307 & 59,4 \\
\hline Não & 59 & 11,4 \\
\hline Ignorado & 29 & 5,6 \\
\hline Não Informado & 122 & 23,6 \\
\hline Total & 517 & 100,0 \\
\hline \multicolumn{3}{|l|}{ Fonte de Informação } \\
\hline Boletim de Ocorrência & 192 & 37,1 \\
\hline Hospital & 128 & 24,8 \\
\hline Família & 29 & 5,6 \\
\hline Instituto de Medicina Legal & 13 & 2,5 \\
\hline Outra fonte & 23 & 4,4 \\
\hline Múltiplas fontes & 3 & 0,6 \\
\hline Ignorado & 24 & 4,6 \\
\hline Não Informado & 105 & 20,3 \\
\hline Total & 517 & 100,0 \\
\hline
\end{tabular}

*Acidente não-de-trânsito: acidentes ocorridos totalmente dentro de garagens, chácaras, canteiros de obras ou áreas não abertas ao público (15).

Fonte: SIM/ Banco de Dados do Sistema Único de Saúde/ Ministério da Saúde, 2012.
. Há uma variação dos tipos de acidente de acordo com o grupo etário envolvido. De 0-11 anos geralmente estão envolvidos transporte pesado ou ônibus e não há envolvimento em colisão com pedestres, animais e veículos a pedal. De 12-17 anos e adultos (18-59 anos), há maior envolvimento de automóveis, carros e caminhonetes. A partir dos 60 anos, a distribuição é equânime entre o grupo de automóveis, caminhonete e caminhões e o de transporte pesado e ônibus.

\section{Discussão}

A maioria dos acidentes decorre de uma série de eventos que aparecem juntos numa sequência $\mathrm{e}$ resultando em numerosas mortes ${ }^{11}$. Anualmente, 180.000 pessoas são hospitalizadas devido a acidentes de tráfego no Brasil ${ }^{12}$, das quais, aproximadamente 37.000 chegam a falecer e destas vítimas fatais $3,8 \%$ são ciclistas.

Apesar de a casuística aparentar ser pequena, o problema com os acidentes envolvendo ciclistas é que, em geral, eles são mais graves, devido a falta de proteção deste tipo de veículo ${ }^{13}$, e por este motivo geram uma maior letalidade.

Vários fatores contribuem para o aumento significativo das injúrias fatais em ciclistas: maior velocidade do veículo antes do impacto, acidentes envolvendo veículos automotores de grande porte, alta velocidade, motorista ou ciclista intoxicado, geralmente por bebidas alcoólicas, bicicletista com idade igual ou superior a 55 anos, intempéries climáticas, iluminação pública deficiente, colisão frontal ${ }^{14} \mathrm{e}$ não utilização de equipamentos de proteção adequados ${ }^{2}$.

No presente estudo, consideraram-se os resultados estaduais, incluindo a divisão espacial em mesorregiões, permitindo evidenciar que muito embora os acidentes ocorram em maior número na Região Metropolitana (composta por apenas 14 municípios e a maior parte da população do estado), o Agreste Pernambucano também é vitimado com bastante frequência por acidentes deste tipo. As causas deste fenômeno carecem de maior pesquisa.

Alguns problemas foram encontrados neste estudo que corroboram os achados de Farias et al. ${ }^{12}$ : a) escassez de pesquisas sobre este assunto, que demonstra a importância de estudos regionais, cujo objetivo é a obtenção de dados que mostrem a prevalência de acidentes de trânsito; $b$ ) falta de clareza na informação sobre a causa de morte. Classificar um acidente como "outros acidentes de tráfego", "acidente desconhecido" ou "causa não 


\section{ESTADO DE PERNAMBUCO: MESORREGIÕES}

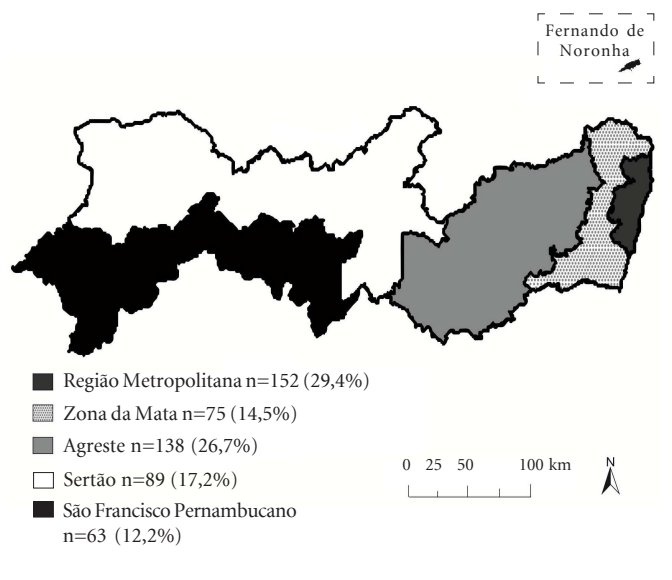

Figura 2. Distribuição espacial das mortes devido a acidentes com ciclistas/bibicletistas, Pernambuco, 2001-2010.

informada" prejudica a entrada de dados no SIM e o diagnóstico preciso deste agravo.

Outra questão é a classificação da causa jurídica de morte, que pode ser: acidente, homicídio ou suicídio. Esta questão independe do preenchimento da certidão de óbito e, por este motivo, não é competência ou responsabilidade do médico legista. Na verdade, esta questão consta em uma área da certidão que só é preenchida para fins epidemiológicos.

Houve outras limitações devido à escolha da fonte de informação e dentre elas pode-se apontar:

. Impossibilidade de se determinar o motivo do deslocamento de bicicleta (lazer, transporte, esporte competitivo ou outros);

. Impossibilidade de se identificar fatores relativos à vítima (uso de equipamentos de proteção obrigatórios, nível de consciência - normal, alterado por uso de substâncias alcóolicas e/ou entorpecentes, comportamentos imprudentes); e,

. Em $28,8 \%$ dos casos, não foi possível identificar situações relativas aos acidentes (veículos ou meios envolvidos, casos de imprudência de uma ou de ambas as partes envolvidas, se ocorreram problemas de sinalização, de iluminação ou de estrutura das vias), pois estes foram catalogados como inespecíficos.
As limitações apontadas sinalizam para a necessidade ou de pesquisas de campo ou de pesquisas usando outras fontes de dados. No que diz respeito ao Sistema de Informação de Mortalidade aponta para a necessidade de se aperfeiçoar o sistema com a inclusão de tais variáveis, posto serem de importância no estabelecimento de políticas públicas adequadas.

Mesmo com estas dificuldades, a escolha por trabalhar com os dados de mortalidade acontece por este indicador fornecer dados mais robustos no campo epidemiológico ${ }^{15}$.

Além disso, a falta ou a divulgação limitada dos dados relativos a este tipo de acidente pode auxiliar na manutenção ou no aumento do número de mortes em acidentes envolvendo bicicletas. A falta de registro de acidentes com bicicleta ocorre porque muitas vezes não envolvem terceiros ou danos à propriedade, portanto, raramente levando ao envolvimento da polícia ${ }^{5}$. No caso específico do Estado de Pernambuco, esta lacuna na informação faz com que campanhas para o emprego da bicicleta como um meio de transporte seguro e eficaz não ocorram, enquanto se promove campanhas eficazes e consideradas inovadoras para o uso de motocicletas e contra a associação de bebida e direção.

Na maioria dos casos, os acidentes de trânsito ocorreram devido a deficiências nas estradas e dos veículos e erros humanos ${ }^{16}$. Entretanto, os benefícios apresentados pela prática do ciclismo (redução da emissão de poluição no ar, melhoria na saúde geral) compensam os riscos envolvidos com a prática da atividade (acidente de trânsito e lesões mais graves, inalação de gases poluentes, etc.). Todavia, para otimizar os benefícios para a sociedade, as políticas de incentivo ao ciclismo devem vir acompanhadas por planejamento de transporte adequado e medidas de segurança ${ }^{13}$.

\section{Conclusões}

O estudo aponta para a urgente necessidade de criação de infraestrutura adequada a esta prática e medidas legais efetivas para prevenir acidentes de trânsito envolvendo bicicletas. Embora os acidentes aconteçam em maior número nos grandes centros urbanos, sua ocorrência com vítimas fatais na maioria dos municípios evidencia que políticas estaduais e municipais devem ser desenvolvidas para minimizar este problema. 
Tabela 3. Cruzamento entre Causa Básica de Morte e características sociodemográficas.

\begin{tabular}{|c|c|c|c|c|c|c|c|c|c|c|}
\hline \multirow[b]{3}{*}{ Variáveis } & \multicolumn{10}{|c|}{ Causa Básica de Morte } \\
\hline & \multicolumn{2}{|c|}{$\mathrm{CPO}$} & \multicolumn{2}{|c|}{ COVP } & \multicolumn{2}{|c|}{ CVM 2-3R } & \multicolumn{2}{|c|}{ CACC } & \multicolumn{2}{|c|}{ CTOP } \\
\hline & $\mathbf{N}$ & $\%$ & $\mathbf{N}$ & $\%$ & $\mathbf{N}$ & $\%$ & $\mathbf{N}$ & $\%$ & $\mathbf{N}$ & $\%$ \\
\hline Gênero & \multicolumn{2}{|c|}{$\mathrm{p}=0,613$} & \multicolumn{2}{|c|}{$\mathrm{p}=0,979$} & \multicolumn{2}{|c|}{$\mathrm{p}=0,890$} & \multicolumn{2}{|c|}{$\mathrm{p}=0,361$} & \multicolumn{2}{|c|}{$\mathrm{p}=0,903$} \\
\hline Masculino & 12 & 92,3 & 13 & 92,9 & 37 & 92,5 & 97 & 87,5 & 84 & 93,3 \\
\hline Feminino & 1 & 7,7 & 1 & 7,5 & 3 & 7,5 & 5 & 12,5 & 6 & 6,7 \\
\hline Total & 13 & 100,0 & 14 & 100,0 & 40 & 100,0 & 102 & 100,0 & 90 & 100,0 \\
\hline Grupo etário & \multicolumn{2}{|c|}{$\mathrm{p}=0,859$} & \multicolumn{2}{|c|}{$\mathrm{p}=0,025$} & \multicolumn{2}{|c|}{$\mathrm{p}=0,391$} & \multicolumn{2}{|c|}{$\mathrm{p}=0,956$} & \multicolumn{2}{|c|}{$\mathrm{p}=0,619$} \\
\hline $0-11$ & 0 & 0,0 & 0 & 0,0 & 1 & 2,5 & 3 & 2,9 & 6 & 6,7 \\
\hline $12-17$ & 0 & 0,0 & 4 & 28,6 & 3 & 7,5 & 8 & 7,8 & 7 & 7,8 \\
\hline $18-24$ & 3 & 23,1 & 1 & 7,1 & 9 & 22,5 & 18 & 17,6 & 13 & 14,4 \\
\hline $25-39$ & 4 & 30,8 & 5 & 35,7 & 7 & 17,5 & 31 & 30,5 & 25 & 27,8 \\
\hline $40-59$ & 4 & 30,8 & 4 & 28,6 & 13 & 32,5 & 28 & 27,5 & 25 & 27,8 \\
\hline $60 \mathrm{ou}+$ & 2 & 15,3 & 0 & 0,0 & 7 & 17,5 & 14 & 13,7 & 14 & 15,5 \\
\hline Total & 13 & 100,0 & 14 & 100,0 & 40 & 100,0 & 102 & 100,0 & 90 & 100,0 \\
\hline Cor & \multicolumn{2}{|c|}{$\mathrm{p}=0,176$} & \multicolumn{2}{|c|}{$\mathrm{p}=0,585$} & \multicolumn{2}{|c|}{$\mathrm{p}=0,963$} & \multicolumn{2}{|c|}{$\mathrm{p}=0,199$} & \multicolumn{2}{|c|}{$\mathrm{p}=0,952$} \\
\hline Branco & 3 & 23,1 & 5 & 35,7 & 7 & 17,5 & 23 & 22,5 & 18 & 20,0 \\
\hline Preto & 2 & 15,4 & 0 & 0,0 & 1 & 2,5 & 3 & 2,9 & 2 & 2,2 \\
\hline Amarelo & 0 & 0,0 & 0 & 0,0 & 0 & 0,0 & 1 & 1,0 & 0 & 0,0 \\
\hline Indígena & 0 & 0,0 & 0 & 0,0 & 0 & 0,0 & 1 & 1,0 & 0 & 0,0 \\
\hline Pardo & 8 & 61,5 & 9 & 64,3 & 31 & 77,5 & 68 & 66,7 & 65 & 72,2 \\
\hline Não informado & 0 & 0,0 & 0 & 0,0 & 1 & 2,5 & 6 & 5,9 & 5 & 5,6 \\
\hline Total & 13 & 100,0 & 14 & 100,0 & 40 & 100,0 & 102 & 100,0 & 90 & 100,0 \\
\hline Estado Civil & \multicolumn{2}{|c|}{$\mathrm{p}=0,836$} & \multicolumn{2}{|c|}{$\mathrm{p}=0,377$} & \multicolumn{2}{|c|}{$\mathrm{p}=0,013$} & $\mathrm{p}=$ & 0,481 & $\mathrm{p}=$ & 0,848 \\
\hline Solteiro & 7 & 53,8 & 7 & 50,0 & 23 & 57,5 & 60 & 58,8 & 48 & 53,3 \\
\hline Casado & 5 & 38,5 & 4 & 28,6 & 12 & 30,0 & 31 & 30,5 & 32 & 35,5 \\
\hline Ignorado & 0 & 0,0 & 3 & 21,4 & 2 & 5,0 & 9 & 8,8 & 6 & 6,7 \\
\hline Não informado & 1 & 7,7 & 0 & 0,0 & 3 & 7,5 & 2 & 1,9 & 4 & 4,4 \\
\hline Total & 13 & 100,0 & 14 & 100,0 & 40 & 100,0 & 102 & 100,0 & 90 & 100,0 \\
\hline Escolaridade & $\mathrm{p}=$ & 0,220 & $\mathrm{p}=$ & 0,555 & $\mathrm{p}=$ & 0,331 & $\mathrm{p}=$ & 0,782 & $\mathrm{p}=$ & 0,869 \\
\hline Nenhum & 0 & 0,0 & 1 & 7,1 & 10 & 25,0 & 13 & 12,7 & 13 & 14,4 \\
\hline 1-3 anos & 3 & 23,1 & 4 & 28,6 & 5 & 12,5 & 16 & 15,7 & 11 & 12,2 \\
\hline 4-7 anos & 2 & 15,3 & 1 & 7,1 & 6 & 15,0 & 19 & 18,7 & 19 & 21,1 \\
\hline $8-11$ anos & 3 & 23,1 & 2 & 14,3 & 3 & 7,5 & 7 & 6,9 & 5 & 5,6 \\
\hline 12 anos ou mais & 0 & 0,0 & 0 & 0,0 & 0 & 0,0 & 1 & 0,9 & 2 & 2,2 \\
\hline Ignorado & 3 & 23,1 & 5 & 35,8 & 12 & 30,0 & 27 & 26,5 & 27 & 30,0 \\
\hline Não informado & 2 & 15,3 & 1 & 7,1 & 4 & 10,0 & 19 & 18,6 & 13 & 14,5 \\
\hline Total & 13 & 100,0 & 14 & 100,0 & 40 & 100,0 & 102 & 100,0 & 90 & 100,0 \\
\hline
\end{tabular}


Tabela 3. continuação

\begin{tabular}{|c|c|c|c|c|c|c|c|c|}
\hline \multirow[b]{3}{*}{ Variáveis } & \multicolumn{8}{|c|}{ Causa Básica de Morte } \\
\hline & \multicolumn{2}{|c|}{ COVNM } & \multicolumn{2}{|c|}{ COFE } & \multicolumn{2}{|c|}{ ANDT } & \multicolumn{2}{|c|}{$\mathrm{CPO}$} \\
\hline & $\mathbf{N}$ & $\%$ & $\mathbf{N}$ & $\%$ & $\mathbf{N}$ & $\%$ & $\mathbf{N}$ & $\%$ \\
\hline Gênero & \multicolumn{2}{|c|}{$\mathrm{p}=0,698$} & \multicolumn{2}{|c|}{$\mathrm{p}=0,021$} & \multicolumn{2}{|c|}{$\mathrm{p}=0,489$} & \multicolumn{2}{|c|}{$\mathrm{p}=0,535$} \\
\hline Masculino & 2 & 100,0 & 10 & 76,9 & 89 & 94,7 & 137 & 91,9 \\
\hline Feminino & 0 & 0,0 & 3 & 23,1 & 5 & 5,3 & 12 & 8,1 \\
\hline Total & 2 & 100,0 & 13 & 100,0 & 94 & 100,0 & 149 & 100,0 \\
\hline Grupo etário & \multicolumn{2}{|c|}{$\mathrm{p}=0,427$} & \multicolumn{2}{|c|}{$\mathrm{p}=0,184$} & \multicolumn{2}{|c|}{$\mathrm{p}=0,797$} & \multicolumn{2}{|c|}{$\mathrm{p}=0,106$} \\
\hline $0-11$ & 0 & 0,0 & 1 & 7,7 & 4 & 4,3 & 6 & 4,0 \\
\hline $12-17$ & 0 & 0,0 & 2 & 15,4 & 6 & 6,4 & 6 & 4,0 \\
\hline $18-24$ & 0 & 0,0 & 4 & 30,8 & 15 & 15,9 & 20 & 13,4 \\
\hline $25-39$ & 0 & 0,0 & 5 & 38,4 & 26 & 27,7 & 60 & 40,3 \\
\hline $40-59$ & 2 & 100,0 & 1 & 7,7 & 33 & 35,1 & 40 & 26,8 \\
\hline $60 \mathrm{ou}+$ & 0 & 0,0 & 0 & 0,0 & 10 & 10,6 & 17 & 11,5 \\
\hline Total & 2 & 100,0 & 13 & 100,0 & 94 & 100,0 & 149 & 100,0 \\
\hline Cor & \multicolumn{2}{|c|}{$\mathrm{p}=0,923$} & \multicolumn{2}{|c|}{$\mathrm{p}=0,973$} & \multicolumn{2}{|c|}{$\mathrm{p}=0,421$} & \multicolumn{2}{|c|}{$\mathrm{p}=0,656$} \\
\hline Branco & 1 & 50,0 & 3 & 23,1 & 12 & 12,8 & 23 & 15,4 \\
\hline Preto & 0 & 0,0 & 0 & 0,0 & 5 & 5,3 & 3 & 2,1 \\
\hline Amarelo & 0 & 0,0 & 0 & 0,0 & 0 & 0,0 & 0 & 0,0 \\
\hline Indígena & 0 & 0,0 & 0 & 0,0 & 0 & 0,0 & 1 & 0,7 \\
\hline Pardo & 1 & 50,0 & 9 & 69,2 & 73 & 77,7 & 113 & 75,8 \\
\hline Não informado & 0 & 0,0 & 1 & 7,7 & 4 & 4,2 & 9 & 6,0 \\
\hline Total & 2 & 100,0 & 13 & 100,0 & 94 & 100,0 & 149 & 100,0 \\
\hline Estado Civil & \multicolumn{2}{|c|}{$\mathrm{p}=0,265$} & \multicolumn{2}{|c|}{$\mathrm{p}=0,965$} & \multicolumn{2}{|c|}{$\mathrm{p}=0,156$} & \multicolumn{2}{|c|}{$\mathrm{p}=0,196$} \\
\hline Solteiro & 1 & 50,0 & 6 & 46,1 & 51 & 54,3 & 84 & 56,4 \\
\hline Casado & 0 & 0,0 & 5 & 38,5 & 24 & 25,5 & 48 & 32,2 \\
\hline Ignorado & 1 & 50,0 & 1 & 7,7 & 13 & 13,8 & 6 & 4,0 \\
\hline Não informado & 0 & 0,0 & 1 & 7,7 & 6 & 6,4 & 11 & 7,4 \\
\hline Total & 2 & 100,0 & 13 & 100,0 & 94 & 100,0 & 149 & 100,0 \\
\hline Escolaridade & \multicolumn{2}{|c|}{$\mathrm{p}=0,805$} & \multicolumn{2}{|c|}{$\mathrm{p}=0,879$} & \multicolumn{2}{|c|}{$\mathrm{p}=0,054$} & \multicolumn{2}{|c|}{$\mathrm{p}=0,383$} \\
\hline Nenhum & 0 & 0,0 & 1 & 7,7 & 10 & 10,6 & 19 & 12,7 \\
\hline 1-3 anos & 0 & 0,0 & 2 & 15,4 & 9 & 9,6 & 32 & 21,5 \\
\hline 4-7 anos & 0 & 0,0 & 4 & 30,8 & 14 & 14,9 & 22 & 14,9 \\
\hline 8-11 anos & 0 & 0,0 & 1 & 7,7 & 4 & 4,2 & 9 & 6,0 \\
\hline 12 anos ou mais & 0 & 0,0 & 0 & 0,0 & 5 & 5,3 & 3 & 2,0 \\
\hline Ignorado & 1 & 50,0 & 3 & 23,0 & 35 & 37,3 & 47 & 31,5 \\
\hline Não informado & 1 & 50,0 & 2 & 15,4 & 17 & 18,1 & 17 & 11,4 \\
\hline Total & 2 & 100,0 & 13 & 100,0 & 94 & 100,0 & 149 & 100,0 \\
\hline
\end{tabular}

CPA: Colisão com Pedestre ou Animal; COVP: Colisão com Outro Veículo a Pedal; CVM2-3R: Colisão com Veículo Motorizado de 2-3 Rodas; CACC: Colisão com Automóvel, Caminhonete ou Caminhão; CTPO: Colisão com Transporte Pesado ou Ônibus; CTVF: Colisão com Trem ou Veículo Ferroviário; COVNM: Colisão com Outro Veículo Não-Motorizado; COFE: Colisão com Objeto Fixo ou Estacionário; ANDT: Acidentes Não-de-Trânsito; AI: Acidente Inespecífico. 


\section{Colaboradores}

PVM Galvão participou da concepção, delineamento, análise e interpretação dos dados, além da redação do artigo; LP Pestana, VM Pestana e MOP Spíndola participaram na redação do artigo e revisão crítica; RIC Campello participou da concepção, delineamento e aprovação da versão a ser publicada; EHA Souza, na qualidade de orientadora da autora principal e, por este motivo, autora correspondente, participou de todas as etapas relativas à pesquisa e à publicação dela resultante.

\section{Referências}

1. World Health Organization (WHO). Global status report on road safety - time for action. Geneva: WHO; 2009.

2. Peden M, Scurfield R, Sllet D, Mohan D, Hyder AA, Jarawan E, Mathers C. World report on road traffic injury prevention. Geneva: WHO; 2004.

3. Brasil. Ministério das Cidades (MC). Secretaria Nacional de Transporte e Mobilidade Urbana. Programa Bicicleta Brasil - Programa Brasileiro de Mobilidade por Bicicleta nas cidades. Brasília: MC; 2007.

4. Özkan S, Akdur O, Ikizceli I, Durukan P, Ipekci A, Sözüer. Bicycle related injuries in adults and children in the anatolian region: analysis of 4 years. JAEM 2012; 11:35-40.

5. Juhra C, Wieskötter B, Chu K, Trost L, Weiss U, Messerschimidt M, Malczyk A, Heckwolf M, Raschke M. Bicycle accidents - Do we only see the tip of the iceberg?: A prospective multi-centre study in a large German city combining medical and police data. Injury 2011: 43(12):2026-34.

6. Andersen LB, Schnohr P, Scroll M, Hein HO. Mortality associated with physical activity in leisure time, at work, in sports and cycling to work. Ugeskr Laeger 2002; 164(11):1501-1506.

7. Pereira CU, Abud LN, Abud FN, Leite RT. Traumatismo cranioencefálico por acidente com bicicleta. Arq Bras Neurocir 2000; 19(2):83-87.

8. Bacchiere G, Gigante DP, Assunção MC. Determinantes e padrões de utilização da bicicleta e acidentes de trânsito sofridos por ciclistas trabalhadores na cidade de Pelotas, Rio Grande do Sul, Brasil. Cad Saude Publica 2005; 21(5):1499-1508.

9. Sebban AC. La complementarité entre levélo et lestransportspublic - De lacohabitation à I'intermodalité [tese]. Aix-em-Provence: Institut d'Aménagement; 2003.
10. Instituto Brasileiro de Geografia e Estatística (IBGE). Pernambuco e suas subdivisões. [página da Internet]. [2012 fev 14]. Disponível em: http://www.ibge. gov.br/estadosat/perfil.php?sigla=pe

11. Ostrom LT, Wilhelmsen CA. Risk assessment: tools, techniques, and their applications. $1^{\text {st }}$ Edition. Idaho: Wiley; 2012.

12. Farias MSJA, Cavalcante HPA, Brandão YST, Barros DTR, Correia DS, Cavalcante JC. Analysis of deaths from traffic accidents in a Brazilian capital. International Journal of Collaborative Research on Internal Medicine \& Public Health 2012; 4(5):670-687.

13. Hartog JJ, Boogaard H, Nijland H, Hoek G. Do the Health Benefits of Cycling Outweigh the Risks? Cien Saude Colet 2011; 16(12):4731-4744.

14. Kim JK, Kim S, Ulfarsson GF, Porrello LA. Bicyclist injury severities in bicycle-motor vehicle accidents. Accid Anal Prev 2007; 39(2):238-251.

15. Rojas-Rueda D, Nazelle A, Tainio M, Nieuwenhuijsen MJ. The health risks and benefits of cycling in urban environments compared with car use: health impact assessment study. BMJ 2011; 343:d4521.

16. Marins L, Queiroz MS. A atualidade dos acidentes de trânsito na era da velocidade: uma visão geral. Cad Saúde Pública 2000; 16(1):7-21.

Artigo apresentado em 30/11/2012

Aprovado em 07/01/2013

Versão final apresentada em 27/03/2013 